\title{
INNOVACIÓN SOCIAL EN LA UE
}

\author{
Javier RAMOS \\ INVESTIGADOR EN EL INSTITUTO COMPLUTENSE DE ESTUDIOS INTERNACIONALES (ICEI) \\ UNIVERSIDAD COMPLUTENSE DE MADRID
}

SUMARIO: I. Introducción. II. Reinventando lo social en el nuevo orden global. III. Tipos de innovación social. IV. Innovación social en la UE. V. Consideración final. VI. Bibliografía.

RESUMEN: Aunque la innovación social se plantea como una alternativa a los modelos de protección social y productivismo social imperantes desde los años $50 \mathrm{O}$ a nuestros días, no está del todo claro qué es la innovación social, cuáles son sus principales potencialidades y cuales sus limitaciones más aparentes. Tampoco está claro si hay una única definición de innovación social, o varias en función de cómo esta se posiciona respecto a elementos clave de la relación Estado-Mercado. En este artículo nos proponemos aportar luz a este debate ofreciendo una definición más integral de innovación social basada en el concepto de eficiencracia.

Palabras Clave: crisis, Estrategia Europa-2020, innovación social, eficiencracia, tipos de innovación social

ABSTRACT: Although social innovation is considered to be an alternative to the models of social protection and social productivism prevailing from the $50 \mathrm{O}$ to our days, it is not entirely clear what social innovation is, neither what its most apparent potentialities and limitations are. It is also not clear whether there is a single definition of social innovation or not, depending on how social innovation is seen with respect to key elements of the State-Market relationship. In this article we propose to shed light to this debate by offering a more comprehensive definition based on the concept of efficiencracy.

KEYWORDS: crisis, The Europe 2020 Estrategy, social innovation, efficiencracy, types of social innovation.

\section{Introducción}

En febrero de 20I3, la Comisión Europea a través del Fondo Social Europeo lanzó el Paquete de Inversión Social para apoyar la implementación de la Estrategia UE-2020. Este

\footnotetext{
${ }^{\mathrm{I}}$ En European Commission, Investing in Social Europe, Luxembourg, Publications Office of the European Union, 2013a, pág. 27, se recoge que en el marco financiero que se extiende entre 2007 y 2013, la proporción de fondos asignados a la innovación social osciló entre el ı\% y el $5 \%$ de la financiación total recibida por cada país. Se estima que más de 2.000 millones de euros de estos fondos se dedicaron a la innovación del sector público y
} 
paquete insta a los Estados miembros a priorizar la inversión social para modernizar sus sistemas de bienestar social y para hacer frente a las consecuencias sociales de la crisis económica y el envejecimiento de la población.

Esa modernización pasa por una re-conceptualización de «lo social» desde una nueva perspectiva: «la innovación social» (IS). Lejos de ser irrelevante, esta nueva perspectiva aspira a ser capaz de adaptar las políticas sociales a la nueva realidad cambiante de un mundo comercialmente más internacionalizado, técnicamente más interconectado, laboralmente más precario y desigual y ambientalmente más degradado como consecuencia del cambio climático.

Esta realidad define de manera distinta los ámbitos de la acción social y los retos a los que se enfrentan. La globalización, el desarrollo tecnológico, la crisis financiera de 2007 y las posteriores políticas de austeridad han creado nuevos espacios de vulnerabilidad que necesitan respuestas distintas en contextos institucionales distintos con distintos actores sociales.

Necesariamente, este conjunto de nuevas necesidades, respuestas y actores sitúan a la IS en una esfera distinta a la que ocuparon los modelos de «protección social» predominantes en la era dorada de los Estados de Bienestar (años 50s a 70 os del siglo pasado) así como los modelos de «productivismo social» predominantes durante la etapa de hegemonía neoliberal (años 8os a nuestros días) ${ }^{2}$.

Esto convierte a la IS en una alternativa recurrente, frecuentemente invocada en ámbitos académicos e institucionales como una solución a las necesidades más apremiantes de la nueva realidad social. Sin embargo, no hay una definición unánime que implique una respuesta única a estos retos y por extensión, no hay un tipo único de IS.

Esta disparidad de enfoques y definiciones vinculadas a un solo concepto hace que la IS pueda correr el riesgo de convertirse en ese grupo de conceptos como arte, democracia o libertad, intrínsecamente «disputables» (contested concepts) en tanto que sometidos a continuas redefiniciones ${ }^{3}$.

Por eso, a pesar de los muchos intentos serios de conciliar una definición, la IS sigue siendo un concepto volátil e híbrido ${ }^{4}$, un quasi-concepto ${ }^{5}$ o un concepto-paraguas ${ }^{6}$ que describe una gama tan amplia de ideas, métodos, programas e iniciativas sociales que

más de 1.000 millones de euros fueron actividades innovadoras destinadas a apoyar el desarrollo de competencias y combatir el desempleo.

${ }^{2}$ Ramos, J., «The EU facing Globalisation and the Financial Crisis: The EU-2020 Strategy and the definitive triumph of Commodification in Europe», in RAMOs \& DEL CAMPO (eds), Austerity and the implementation of the Europe-2020 Strategy: a Reflexion from the South, Berna, Peter Lang Publisher, 2017.

${ }^{3}$ Gallie, W. B., «IX. Essentially Contested Concepts», Proceedings of the Aristotelian Society, 56 (I), I956, págs. I67-I98.

${ }^{4}$ Grimm,R., Fox, C., Baines, S. And Albertson, K., «Social innovation, an answer to contemporary societal challenges? Locating the concept in theory and practice», Innovation: The European Journal of Social Science Research, 26:4, 2013, págs. 436-455.

${ }^{5}$ European Commission, Social Innovation Research in the European Union. Approaches, Findings and Future Directions, Luxembourg, Publications Office of the European Union, 2013b (http://www.net4society.eu/_media/ social_innovation.pdf, última consulta en diciembre de 20I7).

${ }^{6}$ Pue, K. Vandergeest C.And Breznitz, D., «Toward a Theory of Social Innovation», Innovation Policy Lab White Paper, 20I6-о, 2016. 
nunca han conducido al desarrollo de una teoría integral de la poder derivar una teoría social.

No está claro aún que prácticas cumplen criterios de IS y cuáles no, entre otras cosas porque esos criterios son a veces confusos, otras muy generales y casi siempre altamente normativos. Esto hace que, por un lado, un gran número de iniciativas estén utilizando con éxito enfoques de IS aunque no usen este término, mientras que otras iniciativas que utilizan el término no estén en realidad llevando a cabo prácticas de IS ${ }^{7}$.

Este artículo aspira a contribuir a clarificar algunos conceptos clave y posibilitar el desarrollo de una teoría más integrada basada en lo que consideramos los dos componentes principales de la IS que la hacen distinta de la protección social y del productivismo social: la mejora en la eficiencia de la acción social y en su efecto redistributivo y la mayor participación democrática en el diseño, implementación y evaluación de las políticas sociales (eficiencracia) $)^{8}$.

Analizaremos los puntos de conexión e integración de las distintas perspectivas de la IS y también los puntos de discrepancia desde los que analizar la amplia gama de conceptos y reconsideraciones específicas en torno a la IS. Por último interpretaremos la visión que desde la UE se está haciendo de la IS y destacaremos sus principales potencialidades y sus limitaciones más aparentes.

Esta definición de la IS basada en la eficiencracia no sólo es capaz de aglutinar a un número cada vez mayor de definiciones y aproximaciones al fenómeno de la IS, sino que, y esto es lo más importante, ofrece un marco analítico desde el que poder medir de forma precisa los aumentos de la eficiencia y la participación de la IS más allá de las conjeturas teóricas y el sesgo especulativo que domina el análisis dela IS en la actualidad.

\section{Reinventando lo social en el nuevo orden global}

La globalización ha tenido efectos económicos positivos como el aumento del comercio y la riqueza mundial, la creciente integración comercial, el incremento del comercio sur-sur, el aumento del empleo y de la fuerza laboral y una significativa reducción de la pobreza.

Sin embargo estas mejoras se han cimentado sobre una creciente financialización y especulación económica, un aumento insostenible de la deuda pública y privada, el empeoramiento de las condiciones laborales, una disminución del peso de los salarios en la riqueza mundial, un aumento significativo de la desigualdad, el aumento de las migraciones económicas y de los refugiados y un deterioro ambiental irreversible?

\footnotetext{
${ }^{7}$ Millard, J. ET AL, «Social Innovation for Poverty Reduction and Sustainable Development: Some Governance and Policy Perspectives», paper presented at ICEGOV20I6, march I-3, 20I6, Montevideo, Uruguay.

${ }^{8}$ Ramos, J., Crowdfunding and the Role of Managers in Ensuring the Sustainability of Crowdfunding Platforms, Sevilla, Institute for Prospective Technological Studies - European Commision, 20I4 (http://publications.jrc.ec.europa.eu/repository/bitstream/JRC85752/jrc85752.pdf, última consulta en diciembre de 20I7).

${ }^{9}$ RAmos, J., «Globalización y Migraciones: La mercantilización de las personas», en Análisis e Investigaciones, 7, 2016, págs. 39-79.
} 
Esta situación ha generado un intenso proceso de proletarización en las economías emergentes y un proceso de fuerte precarización en las economías centrales en un contexto de creciente desigualdad ${ }^{\text {io }}$, que ha puesto de manifestó las carencias y debilidades de los sistemas de redistribución social en los países más desarrollados ${ }^{\mathrm{II}}$.

La IS surge para paliar esas carencias aceptando la existencia de nuevos espacios de vulnerabilidad vinculados al incremento general de la pobreza y la consolidación de la precariedad laboral desde una perspectiva que combina de manera complementaria eficiencia y democracia (eficiencracia).

En este sentido la IS es el intento de hacer armoniosos conceptos que siempre se presentaron como antagónicos, principalmente la relación entre eficiencia e igualdad ${ }^{12}$ Para ello la IS aporta canales para que la participación democrática mejore la eficiencia de la acción social y, a la vez, para que la eficiencia en la aplicación de políticas sociales anime la participación democrática.

Aunque por razones de concreción no podemos hacer un análisis exhaustivo de los componentes centrales del concepto de eficiencracia ligado a la IS, si podemos al menos señalar sus componentes básicos más importantes:

- Eficiencia

- Reformulación de conceptos, procesos, métodos y prácticas sociales.

- Lanzamiento de nuevos productos y servicios sociales.

- Mejoras en la financiación de la acción social.

- Convergencia de iniciativas sociales dispersas y duplicadas.

- Aprendizaje, replicabilidad, sostenibilidad.

- Carácter práctico y aplicado.

- Nuevas formas de financiación, producción, distribución y consumo de base digital

- Participación democrática:

- Reformulación de actores e ideas involucrados en los procesos de IS que animen procedimientos democráticos para gestionar las relaciones de poder, la toma de decisiones y el liderazgo de estos procesos de innovación.

- Esto se espera que ayude al Intercambio de información, nuevas formas de acción colectiva basadas en la colaboración sobre objetivos comunes y liderazgos compartidos.

- Búsqueda de soluciones que impliquen una transformación de las relaciones sociales.

\footnotetext{
${ }^{10}$ RAmos, J., «The EU facing Globalisation and the Financial Crisis: The EU-2020 Strategy and the definitive triumph of Commodification in Europe», cit..

II OECD, Growing unequal? Income distribution and poverty in OECD countries, 2008 (http://www.oecd.org/social/soc/growingunequalincomedistributionandpovertyinoecdcountries.htm, última consulta en diciembre de 20I7); y OECD, Divided we stand: Why inequality keeps reasing, 20II (http://www.oecd.org/els/soc/dividedwestandwhyinequalitykeepsrising.htm, última consulta en diciembre de 20I7).

${ }^{12}$ OKun, A.M., Equality and Efficiency: the Big Tradeoff, Washington, The Brooking Institution, I975.
} 
- Nueva formas de gobernanza digital: nuevas formas de participación y organización comunitaria de base digital.

- Promoción del emprendedor social en entorno sociales e institucionales sensibles a la innovación social.

- Nuevos canales de empoderamiento

Desde esta perspectiva, la IS es tanto un medio para satisfacer las necesidades sociales como un fin para abordar sus nuevos desafíos. Esto dota a la inclusión social de un nuevo significado hasta convertirla en una nueva categoría conceptual y empírica asentada en la eficiencia y la participación democrática.

La IS hace que la inclusión social pase de ser una condición estática, un umbral de rentas, a un ejercicio interactivo mediante el cual lo individual y lo colectivo se repiensan desde la lógica de la eficiencracia. Esto implica una profunda transformación en la relación entre las partes interesadas.

Como plantean Misuraca y Colombo, la IS intenta actuar como un «game changer», rompiendo con dependencias («path dependencies») que tanto la protección social como el productivismo social imponen en el nuevo contexto de crisis $^{13}$. Como resultado, los servicios de IS impulsados por las necesidades presentes requieren el establecimiento de nuevas relaciones de colaboración y nuevos arreglos institucionales.

Por todo esto, la IS tiene más que ver con la práctica social y los actores implicados que con los fundamentos teóricos y las herramientas técnicas que permiten el desarrollo de nuevas fórmulas y sistemas sociales. La IS es principalmente acción. Una acción orientada a satisfacer las necesidades sociales y a producir un cambio original e innovador que genere una mejora sustancial en la prestación de un servicio social o en la producción de un bien básico.

Se trata de diseñar e implementar participativamente soluciones capaces de resolver una situación de insuficiencia social que las instancias tanto públicas como privadas son incapaces de resolver. En este sentido podríamos hablar de la IS como una actividad especialmente pertinente para situaciones sociales a las que ni el mercado ni el Estado han sido capaces de dar respuesta.

\section{Tipos de innovación social}

A pesar de este esfuerzo conceptual, la definición de la IS sigue sometida a reinterpretaciones desde distintas perspectivas ideológicas y no es posible hablar de IS como si de un concepto único y fácilmente identificable se tratase. Se pueden destacar, al menos tres tipos ideales de IS, que no son puros sino que se mezclan características de cada uno en cualquier sistema económico real:

\footnotetext{
${ }^{13}$ Misuraca, G, and Colombo, C., «ICT-Enabled Social Innovation in Support of Public Sector Reform: the Potential of Integrated Approaches to Social Services Delivery to Promote Social Investment Policies in Europe», paper presented at ICEGOV20I6, march I-3, 20I6, Montevideo, Uruguay.
} 
- IS pro-mercado: Prioriza la Eficiencia sobre la Participación Democrática. Considera que la lógica de la competición propia del mercado es el ámbito natural de la IS y donde mejores resultados puede generar.

- No busca una transformación social sino una mejora de las condiciones competitivas del mercado desde las que favorecer el desarrollo de fórmulas de IS.

- El Emprendedor social y las empresas sociales son en realidad empresarios tradicionales que ven en lo social un nuevo ámbito de negocio. Por eso no sólo ven con recelo la participación pública en asuntos sociales sino que cuestionan formula de IS que aspiren a ser una alternativa al mercado.

- Para compensar algunos aspectos nocivos que su actividad productiva puede generar, esta IS está muy próxima a la lógica y los principios representados en la Responsabilidad Social Corporativa. Con la RSC se busca, por un lado solventar algunos problemas sociales y ambientales concretos, convirtiéndose así en un instrumento funcional de la lógica competitiva, y por otro evitar una mala imagen corporativa vinculada a la precariedad o la contaminación ambiental.

- La Economía de mercado es el modelo de referencia.

- IS anti-mercado: Prioriza el componente democrático sobre el eficiente. Es la IS de quienes lanzas proyectos o emprendimientos sociales que no tienen en el beneficio su razón de ser, sino en la resolución de los problemas sociales concretos que dan sentido a su emprendimiento.

- Consideran que la resolución de problemas sociales concretos legitima y da sentido a sus prácticas económicas por encima del beneficio que estas prácticas pueden generar.

- Busca una verdadera transformación social basada en el potencial político y social de la IS como elemento central de una estrategia de desarrollo alternativa basada en la solidaridad y la equidad, en pos de una sociedad socialmente inclusiva.

- Este tipo de emprendimiento social no ve con recelo al Estado ni su acción pública. Las agencias públicas de protección social y los trabajadores sociales que las gestionan son un actor que interactúa el desarrollo de iniciativas sociales que nacen desde la sociedad civil.

- La Economía Social o Solidaria es el modelo de referencia y donde mejor cabida tiene esta lógica de IS.

- IS post-mercado/(post-Estado): Es crítica tanto con las economías capitalistas de mercado por su efecto negativo sobre la igualdad y la cohesión social como con las economías reguladas y planificadas por las ineficiencias burocráticas del Estado y su carácter instrumental al servicio de intereses oligopolísticos.

- Busca una transformación social desde fórmulas alternativas de financiación, organización, producción, distribución y consumo basadas en la colaboración y el interés mutuo, pero no niega ni la necesidad de fórmulas eficientes y sostenibles para que los proyectos puedan ser sostenibles, ni la participación 
del Estado en el desarrollo y consolidación de los mismos para hacerlos más inclusivos y escalables.

- El modelo en el que se mira la IS post-mercado es el emprendedor integral, altamente vinculado a la lógica del «Triple Balance», esto es el conjunto de valores, retos y procesos necesarios para crear valor económico, social y ecológico a la vez. El impacto de cualquier actividad empresarial es triple. El retorno económico buscado está estrechamente relacionado con el bienestar de las personas y la salud del planeta ${ }^{\mathrm{T}}$.

- Son una forma de proporcionar IS allí donde ni el mercado ni el Estado son capaces de proporcionarla, dando una respuesta a los problemas socialmente nocivos (paro, precariedad, deterioro ambiental etc.) que ni las empresas ni las instituciones públicas están siendo capaces de dar.

- La Economía colaborativa es el modelo de referencia, si bien el carácter netamente pro-mercado de los grandes de la economía colaborativa (Airbnb o Über) han puesto en cuestión la novedad y oportunidad de sus actividades y, sobre todo, de sus estrategias impositivas.

\section{Innovación social en la UE}

Vinculada a la Estrategia Europa-2020, la Comisión Europea (CE) define la IS como una respuesta a los problemas sociales actuales basada en otra forma de producir valor, menos centrada en el beneficio financiero y más en demandas o necesidades reales, como una atractiva premisa para reconsiderar nuestros sistemas de producción y redistribución ${ }^{15}$.

Este enfoque se basa en gran medida en una definición previa de IS aportada por la CE en 20I3 y que resalta los siguientes elementos básicos:

- Nuevas respuestas a las demandas sociales más apremiantes, que afectan al proceso de las interacciones sociales.

- El desarrollo e implementación de nuevas ideas (productos, servicios y modelos) para satisfacer las necesidades sociales y crear nuevas relaciones o colaboraciones sociales.

- La necesidad central de mejorar el bienestar humano.

- El componente netamente social de la IS. Social tanto en sus fines como en sus medios. Son innovaciones que no sólo son buenas para la sociedad en su conjunto, sino que también mejoran la capacidad de acción de las personas ${ }^{\mathrm{i} 6}$.

${ }^{14}$ OGEL, L., «Fundamentos del Triple Balance» (http://www.praxxis.es/es/noticias/noticias/165 y http://www.praxxis.es/es/noticias/noticias/I66, última consulta en ambos en diciembre de 20I7).

${ }^{15}$ European Commission, Social Innovation: A Decade of Changes, Luxembourg, Publications Office of the European Union, 20I4a, pág. 8 (http://ec.europa.eu/DocsRoom/documents/I3403/attachments/I/translations, última consulta en diciembre de 20I7).

${ }^{16}$ European Commission (DG Regional and Urban Policy), Guide to Social Innovation, Luxembourg, Publications Office of the European Union, 20I3c, pág. 6 (http://ec.europa.eu/regional_policy/ sources/docgener/presenta/social_innovation/social_innovation_20I3.pdf, última consulta en diciembre de 20I7). 
La Oficina de Asesores de Política Europea (BEPA) (Comisión Europea, 20Io) también ha argumentado que hay una serie de dimensiones de la innovación social que necesitan ser atendidas o acomodadas dentro de una definición común empleada por la Unión Europea. Según la BEPA, la innovación social tiene como objetivo:

- Satisfacer las demandas sociales de los grupos vulnerables que no se encuentran actualmente en el acuerdo socioeconómico existente.

- Abordar los retos sociales en los que se desdibuja la frontera entre lo «social» y lo «económico».

- Promover un enfoque participativo de la organización social y las interacciones que se centre en el empoderamiento.

Estos objetivos no se consideran mutuamente excluyentes. Las innovaciones que satisfacen las necesidades sociales son también capaces de abordar el desarrollo de nuevas formas de organización social que faciliten la participación y el empoderamiento. Y a su vez estas formas de organización democráticas son capaces de favorecer procesos de innovación más eficientes que son capaces de resolver necesidades sociales no resueltas.

\section{Consideración Final}

Desde esta perspectiva teórica no hay nada objetable a las definiciones de la UE. Sin embargo cuando analizamos el contexto en el que surge y donde se pretende aplicar, la Estrategia Europa-2020, entonces los contenidos de la IS y su aplicación adoptan un sentido muy próximo a lo que hemos definido como «IS pro-mercado».

Esto es así porque aunque la E-2020 se pensó como instrumento de transición productiva hacia modelos más inteligentes, sostenibles e inclusivos, donde la IS podría jugar un papel central, en realidad está subordinada a los requisitos del Pacto de Estabilidad y Crecimiento (Maastricht) y a las políticas de austeridad y devaluación interna impuesta tras la crisis financiera de 2007.

Esto es así desde el lanzamiento de la E-2020 en el año 20IO, pero especialmente en 2014 que es cuando se reconoce sin pudor que los objetivos de empleo y pobreza eran inalcanzables como consecuencia de la crisis financiera y que el objetivo central de la Estrategia es fortalecer la recuperación económica.

En ese contexto, la dimensión social de la E-2020, como sucedió en la Estrategia de Lisboa después del informe Kok (2004), está subordinada a la prioridad de garantizar crecimiento y la creación de empleo. Una vez más se actúa bajo la premisa de que la mejor política social es tener trabajo.

La serie de propuestas sobre empleo, I+D+i, sostenibilidad ambiental, educación y pobreza que conforman el esqueleto de la Estrategia están sometidas a la consolidación de los Paquetes de Austeridad y por tanto la IS queda subordinada a su dimensión más mercantilizadora en la línea «pro-mercado» discutida anteriormente.

Esto sin embargo no anula el potencial de la IS en la UE y la capacidad ciudadana para dotar a la IS de un sentido menos mercantilizado. La apuesta por modelos más 
eficientes y democráticos puede favorecer un tránsito desde los modelos de acción pública dirigidos de «arriba-abajo» típicos de la UE a otros modelos mucho más democráticos e inclusivos que pueden mejorar la eficiencia de las políticas sociales.

\section{Bibliografía}

Bureau of European Policy Advisers (BEPA), Empowering people, driving change: Social innovation in the European Union, Luxembourg, Publications Office of the European Union, 2010 (http://net4society.eu/_media/Social_innovation_europe.pdf, última consulta en diciembre de 20I7)

European Commission, Investing in Social Europe, Luxembourg, Publications Office of the European Union, 2013a.

European Commission, Social Innovation Research in the European Union. Approaches, Findings and Future Directions, Luxembourg, Publications Office of the European Union, 2013b (http://www.net4society.eu/_media/social_innovation.pdf, última consulta en diciembre de 2OI7).

European Commission (DG Regional and Urban Policy), Guide to Social Innovation, Luxembourg, Publications Office of the European Union, 20I3c, pág. 6 (http://ec.europa.eu/ regional_policy/sources/docgener/presenta/social_innovation/social_innovation_20I3.pdf, última consulta en diciembre de 20I7).

European Commission, Social Innovation: A Decade of Changes, Luxembourg, Publications Office of the European Union, 20I4a, pág. 8 (http://ec.europa.eu/DocsRoom/documents/13403/ attachments/I/translations, última consulta en diciembre de 20I7).

European Commission, Promoting Inclusive Growth - European Social Fund thematic paper, Luxembourg, Publications Office of the European Union, 20I4b (http://ec.europa.eu/esf/ BlobServlet?docId=445\&langId=en, última consulta en diciembre de 20I7).

Galdie, W. B., «IX. Essentially Contested Concepts», Proceedings of the Aristotelian Society, 56 (I), I956, págs. 167-198.

Grimm, R., FoX, C., BAines, S. AND AlberTSON, K., «Social innovation, an answer to contemporary societal challenges? Locating the concept in theory and practice», Innovation: The European Journal of Social Science Research, 26:4, 2013, págs. 436-455.

MillaRd, J. ET AL, «Social Innovation for Poverty Reduction and Sustainable Development: Some Governance and Policy Perspectives», paper presented at ICEGOV20I6, march I-3, 20I6, Montevideo, Uruguay.

Misuraca, G, and Colombo, C., «ICT-Enabled Social Innovation in Support of Public Sector Reform: the Potential of Integrated Approaches to Social Services Delivery to Promote Social Investment Policies in Europe», paper presented at ICEGOV20I6, march I-3, 20I6, Montevideo, Uruguay.

OECD, Growing unequal? Income distribution and poverty in OECD countries, 2008 (http://www.oecd.org/social/soc/growingunequalincomedistributionandpovertyinoecdcountri es.htm, última consulta en diciembre de 20I7).

OECD, Divided we stand: Why inequality keeps reasing, $20 \mathrm{II}$ (http://www.oecd.org/els/soc/ dividedwestandwhyinequalitykeepsrising.htm, última consulta en diciembre de 20I7).

OGEL, L., «Fundamentos del Triple Balance», 2017 (http://www.praxxis.es/es/noticias/noticias/165, última consulta en diciembre de $2017 \mathrm{y}$ http://www.praxxis.es/es/noticias/noticias/166, última consulta en diciembre de 20I7).

OKun, A.M., Equality and Efficiency: the Big Tradeoff, Washington, The Brooking Institution, 1975.

Pue, K. VAndergeest C.And BreZnitZ, D., «Toward a Theory of Social Innovation», Innovation Policy Lab White Paper, 2016-ог, 2016.

RAmos, J., Crowdfunding and the Role of Managers in Ensuring the Sustainability of Crowdfunding Platforms, Sevilla, Institute for Prospective Technological Studies - European Commision, 
20I4(http://publications.jrc.ec.europa.eu/repository/bitstream/JRC85752/jrc85752.pdf, última consulta en diciembre de 20I7).

RAmOS, J., «Globalización y Migraciones: La mercantilización de las personas», en Análisis e Investigaciones, 7, 2016, págs. 39-79.

RAmos, J., «The EU facing Globalisation and the Financial Crisis: The EU-2020 Strategy and the definitive triumph of Commodification in Europe», in Ramos \& Del CAmpo (eds), Austerity and the implementation of the Europe-2020 Strategy: a Reflexion from the South, Berna, Peter Lang Publisher, 2017.

ZIEGLER, R., «Social Innovation as a Collaborative Concept», Innovation: The European Journal of Social Science Research, 30:4, 20I7, págs. 388-405. 